\title{
Is the hijab protective? An investigation of body image and related constructs among British Muslim women
}

\author{
Viren Swami ',2*, Jusnara Miah', Nazerine Noorani' and \\ Donna Taylor' \\ 'Department of Psychology, University of Westminster, London, UK, \\ ${ }^{2}$ Department of Psychology, HELP University College, Kuala Lumpur, Malaysia
}

\begin{abstract}
Previous studies have reported equivocal findings concerning the impact of wearing a hijab, or Islamic head- and body-cover, on Muslim women's body image. Here, we sought to examine that impact using a larger sample of Muslim women than has been relied upon and a wider range of body image measures. A total of 587 British Muslim women completed a battery of scales assessing their frequency and conservativeness of hijab use, body image variables, attitudes towards the media and beauty ideals, importance of appearance, and religiosity. Preliminary results indicated that 218 women never used the hijab and 369 women used some form of the hijab at least rarely. Controlling for religiosity, women who wore the hijab had more positive body image, lower internalization of media messages about beauty standards, and placed less importance on appearance than women who did not wear the hijab. Among women who wore the hijab, hijab use significantly predicted weight discrepancy and body appreciation over and above religiosity. These results are discussed in terms of the possible protective impact among British Muslim women of wearing the hijab.
\end{abstract}

Among women in socioeconomically developed settings, anxiety about appearance and negative body image have become so prevalent that they are often considered normative experiences (Cash, 2004; Rodin, Silberstein, \& Striegel-Moore, 1984). This, in turn, has led scholars to seek both putative risk and protective factors that may be targeted in intervention programmes aimed at promoting more positive body image. Thus, for example, studies have identified the possible protective component of such factors as feminist identity (Myers, Ridolfi, Crowther, \& Ciesla, 2012), dance participation (Swami \& Harris, 2012), personality traits (Swami et al., 2013), and sport participation (Swami, Steadman, \& Tovée, 2009).

It is also known that body image is rooted in cultural values and that different cultural groups can have markedly different corporeal experiences (see Anderson-Fye, 2012, for a review). Although much of the work in this area has focused on cross-cultural differences (e.g., Swami, Tovée, \& Harris, 2012; Swami et al., 2010), ethno-cultural affiliation within particular cultures may also offer some women the ability to develop more positive body image. For instance, it has been widely documented that some ethnic minority groups do not internalize Euro-American norms of beauty, such as the thin ideal (e.g., Flynn \&

*Correspondence should be addressed to Viren Swami, Department of Psychology, University of Westminster, 309 Regent Street, London WIB 2UW, UK (email: v.swami@westminster.ac.uk). 
Fitzgibbon, 1998; Rubin, Fitts, \& Becker, 2003), which in turn leads to ethnic differences in the prevalence of negative body image (e.g., Crago, Shisslak, \& Estes, 1996; Pate, Pumariega, Hester, \& Garner, 1992; Swami, Airs, Chouhan, Padilla Leon, \& Towell, 2009).

To date, however, very little research on body image has focused on the impact of religion or religious identity as a distinct construct from ethnic identity (Tolaymat \& Moradi, 2011). This is a notable omission considering findings of positive associations between religious identity and such psychological constructs as life satisfaction (Krause \& Ellison, 2003), optimism and hope (Sethi \& Seligman, 1993), and subjective health status (Karademas, 2010). Moreover, one recent study reported that, among women attending a Christian college, non-anxious God attachment (i.e., a secure and warm relationship with God) was positively associated with body appreciation (Homan \& Cavanaugh, 2013). In explanation, the authors suggested that a non-anxious relationship with God served as a source of acceptance, which reduced a participants' tendency to internalize cultural norms of appearance.

Although this research points to the importance of understanding religious affiliation in relation to body image (see Holman, 2012, for a review), this field of inquiry has focused heavily on Christian populations and largely neglected other traditional faiths, such as Islam. Indeed, scholars have noted the lack of systematic and rigorous psychological research among Muslims generally (see Abu-Raiya \& Pargament, 2011, for a review) and in relation to body image specifically (Mahmud \& Swami, 2010; Tolaymat \& Moradi, 2011). Indeed, there are reasons to expect that Islamic religiousness will be associated with indices of body image. First, and broadly speaking, Islamic religiousness has been associated with measures of well-being, including life satisfaction, positive relations with others, and physical health (e.g., Abu-Raiya, Pargament, Mahoney, \& Stein, 2008; Ghorbani, Watson, \& Shahmohamdi, 2008; Tiliouine, Cummins, \& Davern, 2009).

More specifically, it has been proposed that use of the hijab, or Islamic head- and body-cover, may be a specific within-group factor that is related to Muslim women's corporeal experiences (Mahmud \& Swami, 2010; Swami, 2012; Tolaymat \& Moradi, 2011). Although there are different forms of hijab (Ahmed, 1992; Shirazi, 2001), it is clear that use of the hijab is an important visual identifier (Benn \& Jawad, 2004; Nagel, 2002; Sheridan, 2006) with effects on interpersonal perceptions (see also Unkelbach, Forgas, \& Denson, 2008). In terms of the latter, for example, two studies have shown that, compared to unveiled women, women wearing a hijab were perceived by British men as less attractive and intelligent (Mahmud \& Swami, 2010), as well as less popular, sociable, approachable, and competent (Swami, 2012). How these interpersonal perceptions might affect Muslim women's body image and whether wearing the hijab itself affects their corporeal experiences is less well understood.

On the one hand, it has been suggested that the hijab is a form of sexual objectification (Mernissi, 1987), which serves to maintain gendered forms of oppression (e.g., through seclusion or containment) or a sense of otherness among Muslim women (see Ahmed, 1992). On the other hand, it is also clear that reasons for wearing the hijab in Western countries are highly complex and include many positive elements, such as a desire to attain a sense of identity, to gain respect or esteem, or as part of cultural or religious duty (Droogsma, 2007; Nagel, 2002; Ruby, 2006; Williams \& Vashi, 2007). Moreover, wearing the hijab may confer positive effects on the wearer, in so far as it is seen as a means of averting a sexualized gaze and asserting oneself as a human being rather than a sexualized object (Droogsma, 2007; Ruby, 2006).

To date, however, the extent to which wearing the hijab buffers against negative body image among Muslim women has only been infrequently studied. In one study, 
among Muslim women in Australia, Mussap (2009) reported that modesty of clothing (operationalized as the frequency with which participants wore clothing to cover particular body parts in public) mediated the relationship between religiosity and body dissatisfaction. In another study, it was reported that, in a sample of US women, participants who wore non-Western dress had significantly lower drive for thinness, appearance pressures, and internalization of media messages about appearance (but not body dissatisfaction) compared with counterparts who wore Western dress (Dunkel, Davidson, \& Qurashi, 2010). Most recently, Tolaymat and Moradi (2011) reported that conservativeness of the hijab (measured as greater frequency of use and greater body coverage) was negatively associated with sexual objectification experiences, which in turn had positive relationships with body surveillance, body shape, and eating disorder symptoms.

Despite this growing body of work, a number of issues limit our understanding of the relationships between use of the hijab and body image among Muslim women. First, use of the hijab has not been measured in a consistent manner across studies, which limits the possibility of making cross-study comparisons. It is also not clear that, in some studies (Dunkel et al., 2010; Mussap, 2009), use of the hijab has been measured in a manner that is relevant or sufficiently explicit to be meaningful to Muslim women. Third, in one study (Dunkel et al., 2010), Muslim and non-Muslim women were combined for analysis, which limits conclusions that can be drawn about the use of the hijab specifically. Finally, all previous studies have relied on relatively small sample sizes of Muslim women $(n \leq 118)$.

In consideration of these issues, this study sought to establish the extent to which wearing the hijab confers a protective effect on Muslim women. More specifically, we compared scores on a number of body image measures and related constructs between Muslim women who wear the hijab and Muslim women who do not wear the hijab. The latter may offer a more appropriate comparison group than non-Muslim women, as has been relied upon on previous work (Dunkel et al., 2010; Mussap, 2009). In terms of variables, we examined differences in different but related constructs of body image (i.e., weight discrepancy, body dissatisfaction, body appreciation, and drive for thinness), as well as attitudes towards media messages about appearance and the importance of appearance. Although largely exploratory, we expected that Muslim women who wear the veil would have more positive body image and attitudes towards media messages, as well as place less importance on appearance, than Muslim women who do not wear the veil.

\section{Method}

\section{Participants}

Participants of this study were 587 Muslim women recruited from the community in London, UK. Participants ranged in age from 18 to 70 years $(M=27.08, S D=9.87)$ and in self-reported body mass index (BMI) from 15.56 to $35.84 \mathrm{~kg} \mathrm{~m}^{-2} \quad(M=21.46$, $S D=2.59)$. In terms of marital status, the majority of participants were single $(78.9 \%)$, $12.8 \%$ were married, and the remainder were of some other status. In terms of educational qualifications, $5.4 \%$ had completed minimum secondary education, $17.7 \%$ were still in full-time tertiary education, $75.5 \%$ had an undergraduate degree, and $1.7 \%$ had a postgraduate degree. A number of different ethnic groups were represented in the sample, namely Bengali or Bangladeshi (35.8\%), Pakistani (30.7\%), Indian (10.1\%), Arab 
(4.8\%), or other ethnic groups (18.7\%). All participants self-reported as having been born, and were currently residing, in the United Kingdom.

\section{Measures}

Hijab

We followed Tolaymat and Moradi (2011) in obtaining a 'hijab index' consisting of frequency and conservativeness dimensions. Hijab frequency was measured by asking participants 'How frequently do you wear an Islamic headscarf (e.g., hijab, chador, burqa, etc.)?' with responses provided on a 5-point scale $(1=$ Never, $5=$ Always $)$. Conservativeness of hijab was assessed by asking participants whether they wore seven different types of hijab (niqab, burqa, al-Amira, shayla, khimar, chador, other hijab) varying in conservativeness. Note that, unlike Tolaymat and Moradi (2011), we added a seventh item ('other hijab') to capture possibly omitted forms of head- or body-wear. With the exception of this item, all items on this dimension were presented in both text and pictorial form. Ratings were made on a 7-point scale $(0=$ Never, $6=$ Always $)$. A hijab index was calculated as hijab frequency multiplied by composite hijab conservativeness, which higher scores indicating greater frequency of use of more conservative forms of the hijab. In this study, hijab frequency and conservativeness were significantly correlated $(r=.69, p<.001)$, which is consistent with Tolaymat and Moradi (2011). We report on the distribution of the hijab index data and its validity in the Results.

\section{Weight discrepancy}

To measure actual-ideal weight discrepancy, we used the Photographic Figure Rating Scale (PFRS; Swami, Salem, Furnham, \& Tovée, 2008). The PFRS is a figural rating scale consisting of 10 photographic and standardized images of women in front view. All images are presented in greyscale to minimize any confounding effect of skin tone or perceived ethnicity. The women in the PFRS represent the full range of established BMI categories from emaciated to obese (details are available in Swami et al., 2008). Participants were asked to rate the figure that most closely matched their own body (current) and the figure that they would most like to possess (ideal). All responses were made on a 10-point scale $(1=$ Figure with the lowest BMI, $10=$ Figure with the highest BMI). A measure of actual-ideal weight discrepancy was computed as the difference between unsigned (absolute) current and ideal ratings, such that more positive scores reflect greater weight discrepancy (Swami \& Tovée, 2009). Previous work has shown that scores derived from the PFRS have good patterns of validity and good test-retest reliability after 3 weeks (Swami, Taylor, \& Carvalho, 2011; Swami et al., 2008, 2012).

\section{Body dissatisfaction}

Body dissatisfaction was measured using the Body Dissatisfaction subscale of the Eating Disorder Inventory (EDI-BD; Garner, 2004). This is a 9-item measure of dissatisfaction with one's overall shape and with the size of specific body parts. Responses are made on a 6-point scale ( 1 = Never, 6 = Always $)$ and an overall score was computed as the mean of all nine items following reverse-coding of some items. Higher scores on this measure indicate greater body dissatisfaction. The EDI-BD has been shown to have good psychometric properties (Garner, 2004; Spillane, Boerner, Anderson, \& Smith, 2004). In this study, Cronbach's $\alpha$ for this subscale was .90 . 


\section{Drive for thinness}

To measure drive for thinness, we used the 7-item Drive for Thinness subscale of the EDI (EDI-DT; Garner, 2004), which measures preoccupation with body weight, intense fear of becoming fat, and excessive concern with dieting. Items are rated on a 6-point scale $(1=$ Never, 6 = Always $)$ and an overall score was computed as the mean of all items, with higher scores reflecting greater drive for thinness. The EDI-DT has been shown to have good reliability and validity (Garner, 2004; Spillane et al., 2004) and, in this study, Cronbach's $\alpha$ for this subscale was .87 .

\section{Social physique anxiety}

We measured the degree of anxiety associated with perceived evaluation of one's body or physical appearance using the Social Physique Anxiety Scale (SPAS; Hart, Leary, \& Rajeski, 1989). The SPAS is a 12-item measure in which items are rated on a 5-point scale $(1=$ Not at all like me, 5 = Like me a lot). An overall score was computed as the mean of all items, following reverse-coding of relevant items. Higher scores on this scale reflect greater anxiety associated with perceived evaluation of one's appearance. Hart et al. (1989) reported that the SPAS has adequate construct validity, including indices of internal consistency and test-retest reliability. In this study, Cronbach's $\alpha$ for this scale was .90 .

\section{Sociocultural influences on body image}

Participants were asked to complete three subscales of the Sociocultural Attitudes Toward Appearance Questionnaire-3 (SATAQ-3; Thompson, van den Berg, Roehrig, Guarda, \& Heinberg, 2004). The three subscales in question were Information (the degree to which various media are considered an important source of information about being attractive; nine items), Pressure (feeling pressured by various media to strive for cultural ideals of beauty; seven items), and Internalisation-General (endorsement and acceptance of media messages promulgating unrealistic ideals of beauty and striving towards those ideals; nine items). We omitted the Internalisation-Athlete subscale (endorsement and acceptance of an athletic bodily ideal; five items) because of its lack of relevance to this work. All items are rated on a 5-point scale $(1=$ Definitely disagree, $5=$ Definitely agree $)$ and subscale scores were computed as the mean of items associated with each factor following reverse-coding of relevant items. The SATAQ-3 subscales used in this study have been shown to be internally reliable, with good patterns of convergent and divergent validity (Thompson et al., 2004). In this study, Cronbach's $\alpha$ was .81 for the Information subscale, .82 for the Pressure subscale, and .86 for the Internalisation-General subscale.

\section{Investment in appearance}

The survey included the short-form of the Revised Appearance Schemas Inventory (ASI-R; Cash, Melnyk, \& Hrabosky, 2004), which measures investment in one's appearance along two dimensions, namely Self-Evaluative Salience of Appearance (an individual's self-evaluative salience of their appearance; 12 items) and Motivational Salience of Appearance (the extent to which an individual attends to their appearance and engages in appearance-management behaviours; eight items). All items were rated on a 5-point scale (1 = Disagree, $5=$ Agree $)$ and subscale scores were computed as the mean of items associated with each factor, following reverse-coding of some items. Cash et al. (2004) 
reported that both subscales of the ASI-R had good psychometric properties. In this study, Cronbach's $\alpha$ was .83 for the Self-Evaluative subscale and .88 for the Motivational subscale.

\section{Intrinsic religiosity}

We measured religiosity (i.e., a sincere and intentional integration of religion into one's life) using the 3-item subscale of the Duke Religion Index (Koenig, Patterson, \& Meador, 1997). Items were rated on a 5-point scale $(1=$ Definitely not true, $5=$ Definitely true $)$ and an overall score was computed as the mean of all three items. Higher scores on this subscale reflect greater intrinsic religiosity. The measure has been shown to have adequate psychometric properties (Koenig et al., 1997; Storch, Strawser, \& Storch, 2004) and, in this study, Cronbach's $\alpha$ for this subscale was .88 .

\section{Body mass index}

Participants self-reported their height and weight, which was used to compute BMI as $\mathrm{kg} \mathrm{m}^{-2}$. Self-reported height and weight data have been shown to be very strongly correlated with measured data and are valid for identifying relationships in population studies (Spencer, Appleby, Davey, \& Key, 2002).

\section{Demographics}

Participants provided their demographic details consisting of age, marital status, highest educational qualification, ethnicity, country of birth, and country of residence.

\section{Procedure}

Ethics approval for this study was obtained from the relevant university ethics committee. Three female research assistants recruited participants opportunistically from the research assistants' social networks. Potential participants were invited to take part in a study on religion and health. All participants provided informed consent and completed paper-and-pencil version of the survey in which the order of presentation of the above scales was randomized. Once participants completed the survey, they were given a debriefing sheet containing further information about the study and the contact details of the corresponding author. All participants took part on a voluntary basis and were not remunerated for their time.

\section{Results}

\section{Hijab index}

In their study, Tolaymat and Moradi (2011) did not report on the distribution of their hijab index data, treating the data as having met parametric assumptions for inclusion in path analysis. In our sample, however, it was evident that our hijab index data were highly skewed $(M=5.24, S D=7.37$; skewness $=1.60$, kurtosis $=1.51)$, with a large number of participants reporting that they never wore any form of the hijab. We, therefore, divided our data set into two groups, consisting of participants who reported never wearing any form of the hijab $(n=218)$ and those reporting wearing some form of the hijab at least rarely $(n=369)$. We then conducted between-groups analyses with all included 
Table I. Descriptive statistics (mean $[M]$ and standard deviations [SD]) for all included variables as a function of group (hijab vs. no hijab), and the results of the analyses of covariance ( $d f=584$ ) following the omnibus multivariate analysis of covariance

\begin{tabular}{|c|c|c|c|c|c|c|c|}
\hline \multirow[b]{3}{*}{ Variable } & \multicolumn{4}{|c|}{ Group } & & & \\
\hline & \multicolumn{2}{|c|}{$\begin{array}{l}\text { No hijab } \\
(n=218)\end{array}$} & \multicolumn{2}{|c|}{$\begin{array}{c}\text { Hijab } \\
(n=369)\end{array}$} & \multicolumn{3}{|c|}{$\begin{array}{c}\text { Analysis of covariance } \\
\text { results }\end{array}$} \\
\hline & M & $S D$ & M & $S D$ & $F$ & $p$ & $\eta_{p}^{2}$ \\
\hline Weight discrepancy & 1.64 & 1.25 & 1.33 & 1.10 & 16.81 & $<.001$ & .03 \\
\hline Body appreciation & 3.35 & 0.62 & 3.61 & $0.7 \mathrm{I}$ & 12.29 & $<.001$ & .02 \\
\hline Body dissatisfaction & 3.32 & 0.83 & 3.02 & 0.85 & 6.88 & .009 & .01 \\
\hline Drive for thinness & 3.58 & 1.36 & 2.87 & 1.26 & 33.06 & $<.001$ & .05 \\
\hline Social physique anxiety & 3.26 & 0.81 & 2.92 & 0.81 & 13.47 & $<.001$ & .02 \\
\hline SATAQ-3 information & 3.25 & 0.93 & 2.92 & 0.98 & 11.99 & .001 & .02 \\
\hline SATAQ-3 pressures & 2.85 & 1.02 & 2.40 & 1.10 & 14.79 & $<.001$ & .03 \\
\hline SATAQ-3 internalization-general & 3.09 & 1.05 & 2.43 & I.II & 33.19 & $<.001$ & .05 \\
\hline Self-evaluative salience of appearance & 3.12 & 0.85 & 2.70 & 0.78 & 17.13 & $<.001$ & .03 \\
\hline Motivational salience of appearance & 3.58 & 0.88 & 3.27 & 0.66 & 10.61 & .001 & .02 \\
\hline
\end{tabular}

measures as dependent variables and group (hijab vs. no hijab) as the independent variable. All descriptive data are reported in Table 1.

\section{Preliminary analyses}

A univariate analysis of variance showed that there was no significant between-group difference in participant age (hijab $M=26.95, S D=9.72$; no hijab $M=27.14$, $S D=9.99), \quad F(1,587)=0.05, p=.882, \eta_{\mathrm{p}}{ }^{2}<.01$, or in self-reported BMI (hijab $M=21.61, S D=2.78$; no hijab $M=21.21, S D=2.23), F(1,587)=3.17, p=.075$, $\eta_{\mathrm{p}}{ }^{2}<.01$. On the other hand, participants who wore the hijab had significantly higher intrinsic religiosity scores $(M=4.30, S D=0.79)$ than participants who did not wear the hijab $(M=3.18, S D=2.20), F(1,587)=79.29, p<.001, \eta_{\mathrm{p}}{ }^{2}=.12$. For this reason, we included intrinsic religiosity of a covariate in subsequent analyses.

\section{Between-group differences}

We conducted a multivariate analysis of covariance (MANCOVA) with hijab group as the independent variable and weight discrepancy, body appreciation, body dissatisfaction, drive for thinness, social physique anxiety, sociocultural influences on body image, and investment in appearance as dependent variables. Intrinsic religiosity was entered as a covariate. The overall MANCOVA was significant, $F(10,575)=7.99, p<.001, \eta_{\mathrm{p}}{ }^{2}=.12$. As can be seen in Table 1, compared to participants who did not wear the hijab, participants who wore the hijab had significantly higher body appreciation and significantly lower weight discrepancy, body dissatisfaction, drive for thinness, social physique anxiety, reliance on media as a source of information about appearance ideals, perceived pressure from media sources, internalization of media messages, self-evaluative salience of appearance, and motivational salience of appearance. Religiosity emerged as a significant covariate on weight discrepancy, body appreciation, body dissatisfaction, social physique anxiety, and the two ASI-R subscales $\left(F \mathrm{~s}=4.74-12.95, \eta_{\mathrm{p}}{ }^{2} \leq .03\right)$, but not for any of the remaining variables. 


\section{Inter-scale correlations and multiple regressions}

For participants who wore the hijab only, we examined bivariate correlations between the hijab index and all remaining variables. Results showed that the hijab index was significantly correlated with religiosity $(r=.25, p<.001)$, weight discrepancy $(r=-.10$, $p=.046)$, body appreciation $(r=.21, p<.001)$, social physique anxiety $(r=-.16$, $p=.002)$, using the media as a source of information $(r=-.14, p=.006)$, perceived pressure from the media $(r=-.12, p=.026)$, internalization of media messages $(r=-.20, p<.001)$, and self-evaluative salience of appearance $(r=-.19, p<.001)$, and motivational salience of appearance $(r=-.24, p<.001)$. The hijab index was not significantly correlated with body dissatisfaction, drive for thinness, BMI, or participant age $(r s \leq .07)$.

To examine whether hijab index scores predicted body image, we conducted two multiple regressions with weight discrepancy and body appreciation as the criterion variables, respectively. All remaining variables (social physique anxiety, the SATAQ-3 subscales, the ASI-R subscales, BMI, age, religiosity, and hijab index scores) were entered simultaneously as predictor variables. The regression with weight discrepancy was significant, $F(9,368)=13.48, p<.001$, adj. $R^{2}=.23$, and as can be seen in Table 2 , hijab index scores significantly predicted weight discrepancy once the variance accounted for by other variables had been taken into account. Likewise, the regression with body appreciation was significant, $F(9,368)=72.47, p<.001$, adj. $R^{2}=.64$, with hijab index scores again predicting body appreciation once other variables had been accounted for.

\section{Discussion}

The results of this study suggest that there are significant differences in body image and related constructs between British Muslim women who do and do not wear the hijab. Specifically, we found that participants who wore the hijab generally had more positive body image, were less reliant on media messages about beauty ideals, and placed less importance on appearance than participants who did not wear the hijab. In addition, these effects appeared to be driven by use of the hijab specifically, rather than religiosity, which did not emerge as a significant predictor of weight discrepancy and

Table 2. Results of the multiple regressions with weight discrepancy and body appreciation as criterion variables

\begin{tabular}{|c|c|c|c|c|c|c|c|c|c|c|}
\hline & \multicolumn{5}{|c|}{ Weight discrepancy } & \multicolumn{5}{|c|}{ Body appreciation } \\
\hline & B & SE & $\beta$ & $t$ & $p$ & B & SE & $\beta$ & $t$ & $p$ \\
\hline Social physique anxiety & .51 & .09 & .37 & 5.83 & $<.001$ & -.32 & .04 & -.37 & -8.30 & $<.001$ \\
\hline SATAQ-3 information & .16 & .07 & .14 & 2.19 & .029 & -.01 & .03 & -.02 & -0.42 & .673 \\
\hline SATAQ-3 pressure & .10 & .10 & .10 & 1.06 & .289 & -.04 & .04 & -.06 & -0.92 & .361 \\
\hline $\begin{array}{l}\text { SATAQ-3 } \\
\text { internalization-general }\end{array}$ & .24 & .10 & .24 & 2.29 & .023 & -.20 & .05 & -.31 & -4.31 & $<.00$ \\
\hline $\begin{array}{l}\text { Self-evaluative salience } \\
\text { of appearance }\end{array}$ & .07 & .10 & .05 & 0.70 & .488 & -.20 & .05 & -.20 & -4.30 & $<.00$ \\
\hline $\begin{array}{l}\text { Motivational salience } \\
\text { of appearance }\end{array}$ & .22 & .11 & .13 & 2.11 & .035 & -.14 & .05 & .13 & -3.02 & .00 \\
\hline Body mass index & .13 & .02 & .32 & 6.78 & $<.001$ & -.03 & .01 & -.12 & -3.60 & $<.001$ \\
\hline Duke Religion Index & -.03 & .07 & -.02 & -0.48 & .630 & .01 & .01 & .06 & 1.78 & .07 \\
\hline Hijab index & -.02 & .01 & -.10 & -2.11 & .036 & .07 & .03 & .07 & 2.16 & .03 \\
\hline
\end{tabular}


body appreciation in our regression analyses. Below, we discuss these results in greater detail.

Overall, the present results are consistent with previous work suggesting that women who wear non-Western forms of dress have more positive indices of body image than women who wear Western dress (Dunkel et al., 2010). In this study, we found that participants who wore the hijab had more positive body appreciation, as well as lower weight discrepancy, body dissatisfaction, and drive for thinness than women who did not wear the hijab. In addition, the former group had significantly lower social physique anxiety, more positive attitudes towards media messages, and placed lower importance on appearance than women who did not wear the hijab. It should be noted, however, that the effect sizes of these differences were generally small.

It might thus be concluded that use of the hijab offers Muslim women a small protective effect in terms of their body image. Of course, this is not to suggest that Muslim women who wear the hijab are immune from body image issues (Tolaymat \& Moradi, 2011), but rather that the use of the hijab may act as a buffer against negative body image. For example, women who wore the hijab in this study were significantly less likely to internalize media messages about appearance and less likely to place importance on appearance itself. This, in turn, may result in lower need to attain cultural beauty standards, such as the thin ideal. In addition, it is possible that use of the hijab is associated with lower objectification experiences (Tolaymat \& Moradi, 2011), which in turn means that participants were less likely to self-objectify.

Of course, while use of the hijab itself may offer protection against negative body image, it may also be a proxy for some other, unmeasured protective factor. In this study, we found that use of the hijab was predictive of both weight discrepancy and body appreciation, while intrinsic religiosity was not. Nevertheless, women who wore the hijab did have significantly higher religiosity than women who did not wear the hijab, and religiosity also emerged as a significant covariate for some of our between-group analyses. As such, it might be suggested that there is a complex, and hitherto unexamined, relationship between religiosity, use of the hijab, and body image among Muslim women.

Of course, it must also be reiterated that the effect sizes of between-group differences in this study was very small in most cases. In addition, correlation coefficients between our hijab index scores and measures of interest were weak-to-moderate at best, and the predictive power of the hijab index was small, albeit significant in relation body appreciation and weight discrepancy. Even so, our results may have useful implications for intervention programmes aimed at promoting healthier body image among Muslim women in the West. For example, by identifying those aspects of hijab use that are associated with more positive body appreciation in future studies, it might be possible to isolate factors that can be targeted in intervention programmes.

Taken together, the present findings contribute to a fuller understanding of the role of the hijab in the corporeal experiences of British Muslim women. From a practical point of view, while counsellors and clinicians should not assume that wearing the hijab immunizes Muslim women from negative body image, a fuller understanding of a client's reasons for wearing the hijab may offer important avenues for rejecting prescriptive beauty ideals and promoting a sense of agency in relation to those ideals. Of course, any positive impact of the hijab on subjective experiences of the body may also be tempered by negative interpersonal perceptions (Mahmud \& Swami, 2010; Swami, 2012), highlighting a potentially complex set of outcomes as a result of wearing the hijab.

To fully establish the practical value of our findings, future work should seek replicate and extend the current line of research. For one thing, while we followed Tolaymat and 
Moradi (2011) in obtaining a hijab index, there is currently only limited information on the psychometric properties of this measure. In this study, for example, greater frequency of hijab use was associated with greater religiosity, but further research should seek to more fully establish the validity and reliability of this measure. If it can be shown that this measure if psychometrically sound, researchers would be afforded a consistent manner in which to measure use of the hijab among diverse populations. Future work could also expand on the current design by including potentially important variables that were omitted from this study. For example, Tolaymat and Moradi (2011) suggest that use of the hijab is associated with experiences of sexual objectification, which we did not measure in this study. We also did not examine participants' reasons for wearing the hijab, which might be an important factor in understanding associations between hijab use and body image. In a similar vein, other neglected variables that may have impacted on the present results include the degree of acculturation, self-esteem, extrinsic religiosity, and spirituality. Future work might also improve on the present design by more carefully taking into account the likely complex relationships between hijab use, religiosity, and body image.

As a final point, it is worth reiterating that our results are culturally bound: the lived experiences of Muslim women in Britain are likely very different to that of Muslim women in mainland Europe or North America, and certainly very different to the experiences of women in countries with different cultural, legal, and social prescriptions regarding the hijab. Moreover, we support Tolaymat and Moradi (2011) call for 'social justice efforts that advocate for Muslim women's free choice regarding the hijab'. Accumulating evidence highlights the complexity of understanding the impact of wearing the hijab on women's body image, but also the importance of examining body image and related constructs among relatively neglected populations, such as Muslim women living in the West.

\section{References}

Abu-Raiya, H., \& Pargament, K. I. (2011). Empirically based psychology of Islam: Summary and critique of the literature. Mental Health, Religion, and Culture, 14, 93-115.

Abu-Raiya, H., Pargament, K. I., Mahoney, A., \& Stein, C. (2008). A psychological measure of Islamic religiousness: Development and evidence of reliability and validity. The International Journal for the Psychology of Religion, 18, 291-315.

Ahmed, L. (1992). Women and gender in Islam: Historical roots of a modern debate. New Haven, CT: Yale University Press.

Anderson-Fye, E. P. (2012). Anthropological perspectives on physical appearance and body image. In T. F. Cash (Ed.), Encyclopedia of body image and human appearance (pp. 15-22). London, UK: Elsevier.

Benn, T., \& Jawad, H. (Eds.) (2004). Muslim women in the United Kingdom and beyond: Experiences and images. Boston, MA: Brill.

Cash, T. F. (2004). Body image: Past, present, and future. Body Image, 1, 1-5.

Cash, T. F., Melnyk, S., \& Hrabosky, J. I. (2004). The assessment of body-image investment: An extensive revision of the Appearance Schemas Inventory. International Journal of Eating Disorders, 35, 305-316.

Crago, M., Shisslak, C. M., \& Estes, L. S. (1996). Eating disturbances among American minority groups: A review. International Journal of Eating Disorders, 19, 239-248.

Droogsma, R. A. (2007). Redefining hijab: American Muslim women's standpoints on veiling. Journal of Applied Communication Research, 35, 294-319.

Dunkel, T. M., Davidson, D., \& Qurashi, S. (2010). Body satisfaction and pressure to be thin in younger and older Muslim and non-Muslim women: The role of Western and non-Western dress preferences. Body Image, 7, 56-65. 
Flynn, K. J., \& Fitzgibbon, M. (1998). Body images and obesity risk among Black females: A review of the literature. Annals of Behavioral Medicine, 20, 13-24.

Garner, D. M. (2004). Eating Disorder Inventory-3: Professional manual. Lutz, FL: Psychological Assessment Resources.

Ghorbani, N., Watson, P. J., \& Shahmohamdi, K. (2008). Afterlife Motivation Scale: Correlations with maladjustment and incremental validity in Iranian Muslims. The International Journal for the Psychology of Religion, 18, 22-35.

Hart, E. H., Leary, M. R., \& Rajeski, W. J. (1989). The measurement of social physique anxiety. Journal of Sport and Exercise Psychology, 11, 94-104.

Holman, A. (2012). Religion and the body: An overview of the insertions of religion in the empirical psycho-social research lines on the body. European Journal of Science and Technology, 8, 127-134.

Homan, K. J., \& Cavanaugh, B. N. (2013). Perceived relationship with God fosters positive body image in college women. Journal of Health Psychology. Advance online publication.

Karademas, E. C. (2010). Illness cognitions as a pathway between religiousness and subjective health in chronic cardiac patients. Journal of Health Psychology, 15, 239-275.

Koenig, H., Patterson, G., \& Meador, K. (1997). Religion Index for psychiatric research: A 5-item measure for use in health outcomes studies. American Journal of Psychiatry, 154, 855.

Krause, N., \& Ellison, C. G. (2003). Forgiveness by God, forgiveness of others, and psychological well-being in late life. Journal for the Scientific Study of Religion, 42, 77-93.

Mahmud, Y., \& Swami, V. (2010). The influence of the bijab (Islamic head-cover) on perceptions of women's attractiveness and intelligence. Body Image, 7, 90-93.

Mernissi, F. (1987). The veil and the male elite: A feminist interpretation of women's rights in Islam. New York, NY: Basic Books.

Mussap, A. J. (2009). Strength of faith and body image in Muslim and non-Muslim women. Mental Health, Religion, and Culture, 12, 121-127.

Myers, T. A., Ridolfi, D. R., Crowther, J. H., \& Ciesla, J. A. (2012). The impact of appearance-focused social comparison on body image disturbance in the naturalistic environment: The roles of thin-ideal internalization and feminist beliefs. Body Image, 9, 342-351.

Nagel, C. (2002). Constructing difference and sameness: The politics of assimilation in London's Arab communities. Ethnic and Racial Studies, 25, 258-287.

Pate, J. E., Pumariega, A. J., Hester, C., \& Garner, D. M. (1992). Cross-cultural patterns in eating disorders: A review. Journal of the American Academy of Child and Adolescent Psychiatry, 31, 802-808.

Rodin, J., Silberstein, L., \& Striegel-Moore, R. (1984). Women and weight: A normative discontent. Nebraska Symposium on Motivation, 32, 267-307.

Rubin, L. R., Fitts, M. L., \& Becker, A. E. (2003). 'Whatever feels good in my soul': Body ethics and esthetics among African American and Latina women. Culture, Medicine, and Psychiatry, 27, 49-75.

Ruby, T. F. (2006). Listening to the voices of hijab. Women's Studies International Forum, 29, $54-66$.

Sethi, S., \& Seligman, M. E. P. (1993). Optimism and fundamentalism. Psychological Science, 4, 256-259.

Sheridan, L. P. (2006). Islamophobia pre- and post-September 11th, 2001. Journal of Interpersonal Violence, 21, 317-336.

Shirazi, F. (2001). The veil unveiled: The hijab in modern culture. Gainesville, FL: University Press of Florida.

Spencer, E. A., Appleby, P. N., Davey, G. K., \& Key, T. J. (2002). Validity of self-reported height and weight in 4808 EPIC-Oxford participants. Public Health Nutrition, 5, 561-565.

Spillane, N. S., Boerner, L. M., Anderson, K. G., \& Smith, G. T. (2004). Comparability of the Eating Disorder Inventory-2 between women and men. Assessment, 11, 85-93.

Storch, E. A., Strawser, M. S., \& Storch, J. B. (2004). Two-week test-retest reliability of the Duke Religion Index. Psychological Reports, 94, 993-994. 
Swami, V. (2012). The influence of the bijab (Islamic head-cover) on interpersonal judgments of women: Replication and extension. In J. Marich (Ed.), Psychology of women (pp. 128-140). Hauppauge, NY: Nova Science Publishers.

Swami, V., Airs, N., Chouhan, B., Padilla Leon, M. A., \& Towell, T. (2009). Are there ethnic differences in positive body image among female British undergraduates? European Psychologist, 14, 288-296.

Swami, V., Frederick, D. A., Aavik, T., Alcalay, L., Allik, J., Anderson, D., ... Zivcic-Becirevic, I. (2010). Body weight ideals and body dissatisfaction in 26 countries across 10 world regions: Results of the International Body Project I. Personality and Social Psychology Bulletin, 36, 309-325.

Swami, V., \& Harris, A. S. (2012). Dancing toward positive body image? Examining body-related constructs with ballet and contemporary dancers at different levels. American Journal of Dance Therapy, 34, 39-52.

Swami, V., Salem, N., Furnham, A., \& Tovée, M. J. (2008). Initial examination of the validity and reliability of the female Photographic Figure Rating Scale for body image assessment. Personality and Individual Differences, 44, 1752-1761.

Swami, V., Steadman, L., \& Tovée, M. J. (2009). A comparison of body size ideals, body dissatisfaction, and media influence between female track athletes, martial artists, and non-athletes. Psychology of Sport and Exercise, 10, 609-614.

Swami, V., Stieger, S., Harris, A. M., Nader, I. W., Pietschnig, J., Voracek, M., \& Tovée, M. J. (2012). Further investigation of the validity and reliability of the Photographic Figure Rating Scale for body image assessment. Journal of Personality Assessment, 94, 404-409.

Swami, V., Taylor, R., \& Carvalho, C. (2011). Body dissatisfaction assessed by the Photographic Figure Rating Scale is associated with sociocultural, personality, and media influences. Scandinavian Journal of Psychology, 52, 57-63.

Swami, V., Tovée, M. J., \& Harris, A. S. (2012). An examination of ethnic differences in actual-ideal body weight discrepancy and its correlates in a sample of Malaysian women. International Journal of Culture and Mental Health. Advance online publication.

Swami, V., Tovée, M. J. (2009). A comparison of body dissatisfaction, body appreciation, and media influences between street-dancers and non-dancers. Body Image, 6, 304-307.

Swami, V., Tran, U. S., Hoffmann Brooks, L., Kanaan, L., Luesse, E.-M., Nader, I. W., ... Voracek, M. (2013). Body image and personality: Associations between the Big Five Personality Factors, actual-ideal weight discrepancy, and body appreciation. Scandinavian Journal of Psychology, 54, 146-151.

Thompson, J. K., van den Berg, P., Roehrig, M., Guarda, M. S., \& Heinberg, L. J. (2004). The sociocultural attitudes towards appearance scale-3 (SATAQ-3): Development and validation. International Journal of Eating Disorders, 35, 293-304.

Tiliouine, H., Cummins, R. A., \& Davern, M. (2009). Islamic religiosity, subjective well-being and health. Mental Health, Religion, and Culture, 12, 55-74.

Tolaymat, L. D., \& Moradi, B. (2011). US Muslim women and body image: Links among objectification theory constructs and the hijab. Journal of Counseling Psychology, 58, 383-392.

Unkelbach, C., Forgas, J. P., \& Denson, T. F. (2008). The turban effect: The influence of Muslim headgear and induced affect on aggressive responses in the shooter bias paradigm. Journal of Experimental Social Psychology, 44, 1409-1413.

Williams, R. H., \& Vashi, G. (2007). Hijab and American Muslim women: Creating the space for autonomous selves. Sociology of Religion, 68, 269-287.

Received I I April 2013; revised version received II June 2013 